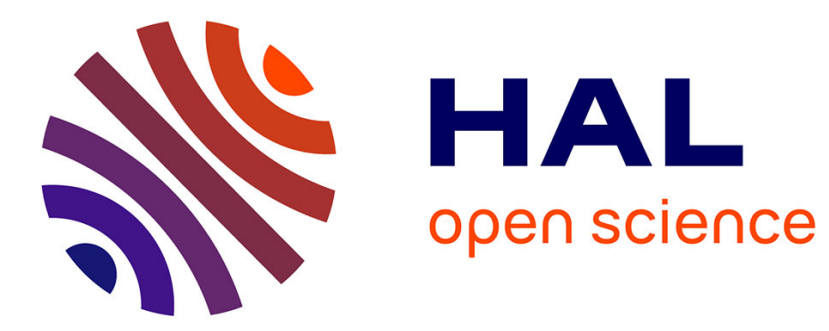

\title{
On the Impact of Link Layer Retransmissions on TCP for Aeronautical Communications
}

Nicolas Kuhn, Nicolas van Wambeke, Mathieu Gineste, Benjamin Gadat, Emmanuel Lochin, Jérôme Lacan

\section{- To cite this version:}

Nicolas Kuhn, Nicolas van Wambeke, Mathieu Gineste, Benjamin Gadat, Emmanuel Lochin, et al.. On the Impact of Link Layer Retransmissions on TCP for Aeronautical Communications. 5th International Conference on Personal Satellite Services - PSATS 2013, Jun 2013, Toulouse, France. pp.38-48, 10.1007/978-3-319-02762-3_4. hal-02552535

\section{HAL Id: hal-02552535 \\ https://hal.science/hal-02552535}

Submitted on 23 Apr 2020

HAL is a multi-disciplinary open access archive for the deposit and dissemination of scientific research documents, whether they are published or not. The documents may come from teaching and research institutions in France or abroad, or from public or private research centers.
L'archive ouverte pluridisciplinaire HAL, est destinée au dépôt et à la diffusion de documents scientifiques de niveau recherche, publiés ou non, émanant des établissements d'enseignement et de recherche français ou étrangers, des laboratoires publics ou privés. 


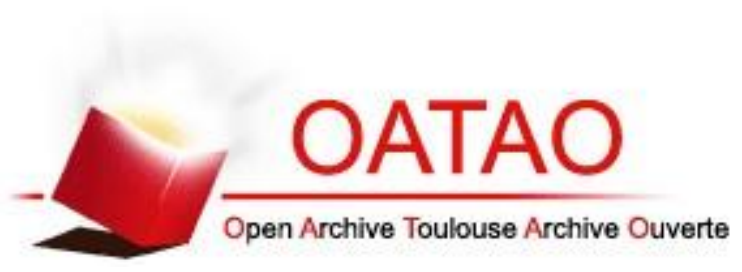

\section{Open Archive Toulouse Archive Ouverte (OATAO)}

OATAO is an open access repository that collects the work of Toulouse researchers and makes it freely available over the web where possible.

This is an author-deposited version published in: http://oatao.univ-toulouse.fr/ Eprints ID: 9205

To cite this document: Kuhn, Nicolas and Van Wambeke, Nicolas and Gineste, Mathieu and Gadat, Benjamin and Lochin, Emmanuel and Lacan, Jérôme On the Impact of Link Layer Retransmissions on TCP for Aeronautical Communications. (2013) In: 5th International Conference on Personal Satellite Services - PSATS 2013, Toulouse, France, 27-28 Jun 2013.

Any correspondence concerning this service should be sent to the repository administrator: staff-oatao@inp-toulouse.fr 


\title{
On the Impact of Link Layer Retransmissions on TCP for Aeronautical Communications
}

\author{
Nicolas Kuhn ${ }^{1,2}$, Nicolas Van Wambeke ${ }^{3}$, Mathieu Gineste ${ }^{3}$, Benjamin Gadat ${ }^{3}$, \\ Emmanuel Lochin ${ }^{2}$, and Jérôme Lacan ${ }^{2}$ \\ 1 NICTA, Sydney, Australia (firstname.lastname@nicta.com.au) \\ ${ }^{2}$ University of Toulouse, ISAE, TeSA, Toulouse, France (firstname.lastname@isae.fr) \\ 3 Thales Alenia Space (TAS), Toulouse, France \\ (fistname.lastname@thalesaleniaspace.com)
}

\begin{abstract}
In this article, we evaluate the impact of link layer retransmissions on the performance of TCP in the context of aeronautical communications. We present the architecture of aeronautical networks, which is manly driven by an important channel access delay, and the various retransmission strategies that can be implemented at both link and transport layers. We consider a worst case scenario to illustrate the benefits provided by the ARQ scheme at the link layer in terms of transmission delay. We evaluate the trade-off between allowing a fast data transmission and a low usage of satellite capacity by adjusting link layer parameters.
\end{abstract}

Key words: aeronautical communications, ats/aoc services,retransmissions strategies,tcp,linklayer

\section{Introduction}

According to recent evolutions in the aeronautical communications domain, advanced safety aeronautical communications are composed by two kinds of service: Air Traffic Services (ATS) and Air Operations Control (AOC), most of these novel services being supported by the SWIM communication paradigm. The goal of such services is to ensure safety and regulate the needs of aeronautical services through the use of binary communication, i.e. transmitting messages between pilots and control centers, instead of actual voice-to-voice communication. ATS/AOC services are small and sporadic, making their transmission over satellite links of interest. In the context of aeronautical communications, the transmission of ATS/AOC services data might be critical and some applications require a reliable transmission with important delay constraints for $95 \%$ of the application data.

When channel codes implemented at the physical layer cannot rebuild packets and forward them to the link layer, retransmissions can be introduced to overcome this problem. The multiple retransmission at different layers might decrease the transmission delay and ensure the reliability of the transmission. However, the satellite resource is expensive and must be equally shared among the users. 
In this article, we illustrate the trade-off between (1) increasing the reliability and decreasing the transmission delay by introducing link layer retransmissions and (2) using more satellite link capacity. We propose simulations in NS-2 conjointly with a realistic model for the plane-satellite channel. We assess the performance of TCP with and without link layer retransmissions on the return link of the plane in terms of transmission delay and capacity utilization.

The rest of the article is organized as follows: in Section 2, we present the aeronautical communication networks and retransmission strategies that can be introduced at different layers. In Section 3, we present the tools used to simulate the whole protocol stack of elements of such complex network. We consider specific scenarios detailed in Section 4. We present and comment the results of the simulations in Section 5 and then conclude this study in Section 6 .

\section{Link and transport layers retransmissions in aeronautical communications}

In this section, we present the aeronautical communications network in terms of structure and traffic carried out. We detail the different delays encountered during the transmission of packets. We list available retransmission strategies specifying at which level they can occur.

\subsection{Satellite, plane and gateway}

In Figure 1, we present the network and highlight the difference between up (also called forward) and down (also called return) links.

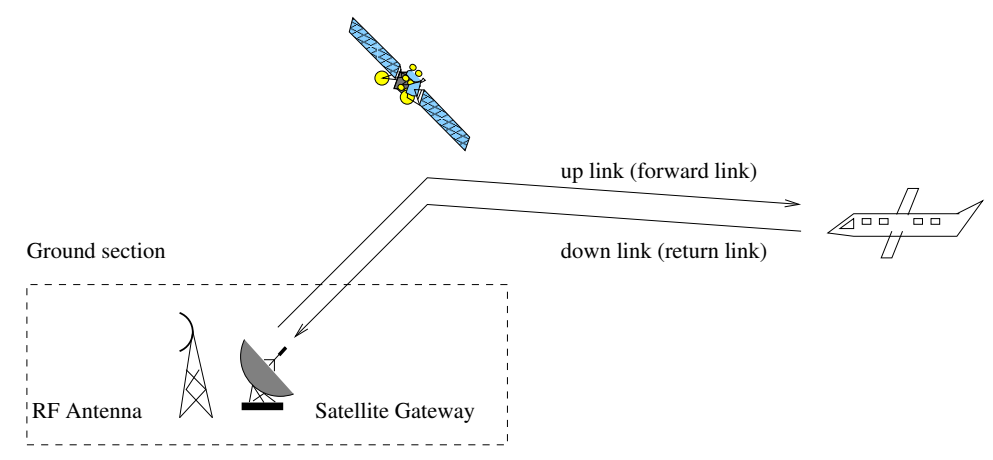

Fig. 1. Plane, satellite and satellite gateway

On the return link (from the plane to the satellite gateway), the average useful throughput is $260 \mathrm{bps}$, which is enough to transmit the small and sporadic data exchanges required by ATC and AOC services. Each application has specific 
requirements in terms of capacity, delay constraint (for $95 \%$ of the packets) or availability ${ }^{1}$. The bounds of these requirements are: (1) the size of data packets is ranging from 70 to 2500 bytes; (2) the inter-packet time for each application varies from $1000 \mathrm{~s}$ to $3000 \mathrm{~s}$; (3) some applications might have delay constraints.

\subsection{Channel access delays}

The network presented in Section 2.1 is complex due to important delays and medium shared between an important number of users. As a result, we present in this section how we model the channel access delays.

The time needed by an applicative data packet to be transmitted from the client to the server (denoted $T_{\text {data }}$ ) is the sum of the propagation delay (denoted $\left.T_{\text {prop }}\right)$, the channel access delay $\left(T_{a c c}\right)$ and retransmission delays $\left(T_{r e t}\right)$ :

$$
T_{\text {data }}=T_{\text {prop }}+T_{a c c}+T_{\text {ret }}
$$

The propagation time is set to $T_{\text {prop }}=250 \mathrm{~ms}$, which is a standard value for satellite transmissions.

\begin{tabular}{ccc}
$\begin{array}{c}\text { Forward } \\
\text { link }\end{array}$ & $\begin{array}{c}\text { Return } \\
\text { link }\end{array}$ \\
\hline Data packets $\in$ & {$[10 ; 100] \in[500 ; 1300]$} \\
TCP Ack. & 55 & 900
\end{tabular}

Table 1. Channel access delay (in $\mathrm{ms}$ )

We detail in Table 1 the values of $T_{a c c}$ for both up and return link.

Forward link

At the physical layer of the satellite gateway, the transmission is scheduled by frames. The important size of the antenna enables the gateway to transmit frames of $90 \mathrm{~ms}$ gathering data for different users. Depending on when the data packet reaches the gateway (will the packet be sent straight away or after $90 \mathrm{~ms}$ ) and considering the $10 \mathrm{~ms}$ necessary for the processing of the frame, we explain the value presented in Table 1 for the forward link. We choose an average delay of $55 \mathrm{~ms}$ for the TCP acknowledgements. The channel access delay can be up to $100 \mathrm{~ms}$ : as a result, it cannot be neglected compared to the propagation delay for both acknowledgements and data packets.

Return link

In order to obtain capacity, a user terminal on the plane requests for capacity following DAMA VBDC access method. Indeed, the medium is fairly shared between the users as the satellite gateway manages to allow dedicated slots (in terms of transmission date and frequency) for each user. As a result, on

\footnotetext{
${ }^{1}$ Unfortunately, this list is confidential and cannot be communicated.
} 
the return link, we consider a delay for the request to be sent to the gateway (between $0 \mathrm{~ms}$ and $800 \mathrm{~ms}$ ), propagation delay for the transmission of the capacity request from the plane to the gateway $(250 \mathrm{~ms})$, propagation delay for the resource allocation plan from the gateway to the users $(250 \mathrm{~ms})$. We neglect the time needed by the gateway to compute this plan which is very low compared to the propagation delays. We consider an average delay of $900 \mathrm{~ms}$ for the TCP acknowledgments.

\subsection{Link and transport layers retransmissions}

The coding schemes introduced at the physical layer might not be suitable to recover the data when the signal-to-noise ratio is low. As a result, we present in this section the retransmissions schemes introduced at both link and transport layers to ensure a reliable transmission.

At the link layer, there are different retransmission techniques which are presented in [?, ?]. We denote by LLDU the Link Layer Data Unit. Among the different existing techniques, we focus on the two following:

Automatic Repeat reQuest (ARQ)

Automatic Repeat reQuest family can be defined by a subset of retransmission strategies (Stop-and-Wait ARQ, Go-Back-N ARQ or Selective-Repeat ARQ). We consider here SR-ARQ mechanism at the link layer level: it consists in the retransmission of the LLDUs that have been lost during the transmission. We denote SR-ARQ by ARQ.

HARQ

Forward Error Correction (FEC) is a scheme where the sender sends a combination of data and repair LLDUs. Let $N_{D}$ (resp. $N_{R}$ ) be the number of data (resp. repair) LLDUs and $N=N_{D}+N_{R}$. The process to recover data LLDUs is successful if at least $N_{D}$ LLDUs are received, otherwise (if the number of erasures is strictly greater than $N_{R}$ ) no correction is possible. The FEC scheme does not enable the retransmission of LLDUs. Hybrid-Automatic Repeat reQuest (HARQ) mechanism is a combination of the FEC and ARQ mechanisms previously described: after the first transmission of a FEC block, including data and repair LLDUs, HARQ allows the sender to transmit additional repair LLDUs when a recovery is not possible at the receiver side. In other words, if no correction is possible, the transmission of additional repair LLDUs is requested by the receiver. Also, if the receiver requests $M$ LLDU, HARQ can send $M+M^{\prime}$, where $M^{\prime}$ is the number of supplementary LLDU that can be transmitted, to potentially reduce the number of retransmissions. In the rest of this article, we consider that HARQ does not transmit a first block FEC but still only transmits repair packets to recover the useful data (i.e., $\left.N_{R}=0\right)$.

At the transport layer, reliable Transmission Control Protocol (TCP) [?] is considered for on-board applications executing in a SWIM like context while the no retransmission User Datagram Protocol (UDP) [?] is considered for COCR like traffic. There are different variants of TCP [?]. Most of them mostly adapt 
the congestion window size in the congestion avoidance phase, however, based on the application sizes detailed in Section 2.1 and the requirements in terms of delay, we focus on TCP New Reno [?] to model the retransmission behaviour as its RFC describes the currently base-line version for TCP. Due to the inter-packet delays considered, TCP never exits the slow-start phase, making the choice for different congestion avoidance mechanism irrelevant.

\subsection{Discussion}

For safety reasons, reliability is compulsory in both kind of environments and different retransmission strategies can be introduced at both link and transport layers (Section 2.3). The transmission delay (including channel access) of retransmissions can be important for both layers (Section 2.2) and some applications have delay constraints (Section 2.1). Moreover, if not properly tuned, introducing retransmissions at multiple layers can lead to counter productive interactions, highly increasing the use of the limited satellite capacity.

In Sections 3,4 and 5, we argue that a trade-off must be found between (1) introducing link layer retransmissions and reducing the transmission delay by using FEC that uses more capacity and (2) no link layer retransmissions, thus leading to a higher end to end transmission delay but using less capacity.

We denote by $A$ the set of applications of each plane, $P$ the set of planes, $D$ one data packet, $C(D)$ and $T_{\text {data }}(D)$ the exploited capacity and transmission delay of $D$, and $T_{M A X}(a)$ the maximum transmission delay authorized for the application $a \in A$.

$$
\forall a \in A, \forall p \in P, \min \left(C(D), T_{\text {data }}(D)\right)
$$

Equation (1) expresses the problem we seek to optimize with the constraint:

$$
T_{\text {prop }}(D)+T_{\text {acc }}(D)<T_{\text {data }}(D)<T_{M A X}(a),
$$

i.e.

$$
T_{\text {ret }}(D)<T_{M A X}(a)-T_{\text {prop }}-T_{\text {acc }}
$$

The objective is thus to adapt the retransmission strategies to reduce $T_{\text {ret }}(D)$ and $C(D)$ for each data packet $D$.

\section{Simulation}

In this section, we present the model and simulation of the network.

The work presented herein uses TDM simulators from $\mathrm{CNES}^{2}$. This simulator takes into account realistic satellite links characteristics, such as satellite orbits or recent correcting codes to generate physical layer traces [?]. As a result,

\footnotetext{
${ }^{2}$ CNES is the french government agency responsible for shaping and implementing France's space policy in Europe, see http://www.cnes.fr/.
} 
each packet transmitted by the physical-layer is characterized by an transmission timestamp and a decoding time. Additionally, its probability to be lost is determined by the physical layer codes and the model of the channel used in the simulation scenario.

Based on the physical layer traces, an simulation of the ARQ or HARQ schemes effects have been derived by the use of the Trace Manager Tool (TMT) [?] that produces link layer traces. These link layer traces are then loaded into the NS-2 network simulation, extended with a Cross-Layer InFormation Tool (CLIFT) [?] to schedule the transmission of applicative packets through the protocol stack (particularly using a specific transport layer) and simulating the behavior of the link and physical layers.

Therefore, with these tools, it is possible to simulate the full protocol stack including link layer FEC, ARQ and HARQ mechanisms to conduct realistic simulations in order to evaluate the impact of retransmissions at the link layer on end to end performances.

\section{Scenario}

In this section, we present the different retransmissions approaches, the traffic and the metrics that we consider to assess the impact of retransmissions at the link layer when the plane transmits data to the satellite gateway.

\section{Retransmissions}

As we detail in Section 2.3, retransmissions can be introduced at different layers of the protocol stack. For compatibility with the SWIM architectures, we argue that the transport layer protocol should be TCP [?]. As a result, we consider the combination of 3 different configuration by varying the link layer reliability mechanism configuration: no retransmission scheme, ARQ at the link layer or HARQ at the link layer.

Traffic

The traffic is generated with exchanges taking place between a plane and a ground system through a satellite gateway (i.e., no air to air communication considered). Applications transmit packets of various sizes $(\epsilon$ [70;2500] bytes), depending the content carried out and the inter-packet time is different for each application $(\in[1000 ; 3000] \mathrm{s})$ (more details can be found in Section 2.1). We consider that there is one TCP connection for each application executing on-board.

Metrics

The transmission of data at the application level is ensured by a reliable transport layer protocol. Therefore, for each $E b / N 0 \in(1.05 d B ; 1.3 d B ; 1.8 d B)$ (i.e. LER (Link layer data unit Error Ratio) $\in 2.3 e^{-1} ; 6 e^{-2} ; 2 e^{-4}$ ) and for each applicative packet size, we measure the transmission delay for $95 \%$ of the packets and the mean transmission delay as most applications are delay-sensitive. We also measure the effective coding ratio (defined by the ratio between the applicative data byte transmitted and the total number 
of byte transmitted on the air interface) to assess the impact of link layer retransmissions on the usage of the available capacity.

\section{Results}

In this section, we present the metrics of the simulations defined in Section 4 . We focus on the benefits that ARQ can provide when used together with TCP and illustrate a worst-case scenario where HARQ is of interest compared to ARQ.

\subsection{On the benefits of ARQ on TCP performance}

In this subsection, we present the evolution of the metrics when $E b / N 0 \in$ $(1.05 d B ; 1.3 d B ; 1.8 d B)$. We plot in Figure 2, the mean delay for the transmission of one packet, in Figure 3, the transmission delay of $95 \%$ of the packets and in Figure 4, the effective coding ratio.

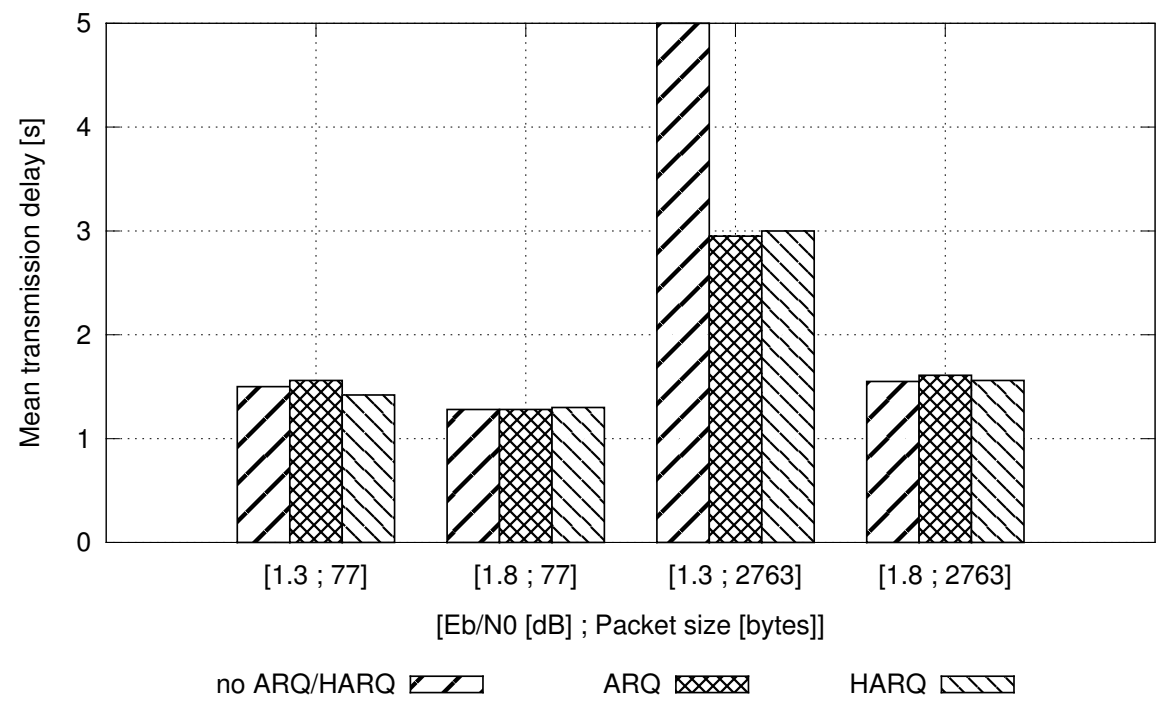

Fig. 2. Mean delay

In Figures 2 and 3, we can see that the benefits provided by ARQ at the link layer is not clear in most of the cases. Indeed, as it can be seen, the use of ARQ brings absolutely no benefit for most of the simulations. However, we can see that when the size of the application packets is high (i.e. 2763 bytes) the average delay necessary to transmit a packet is more than $5 \mathrm{~s}$ (around $200 \mathrm{~s}$, note that we do not represented this value in order to let the figure readable). We argue 


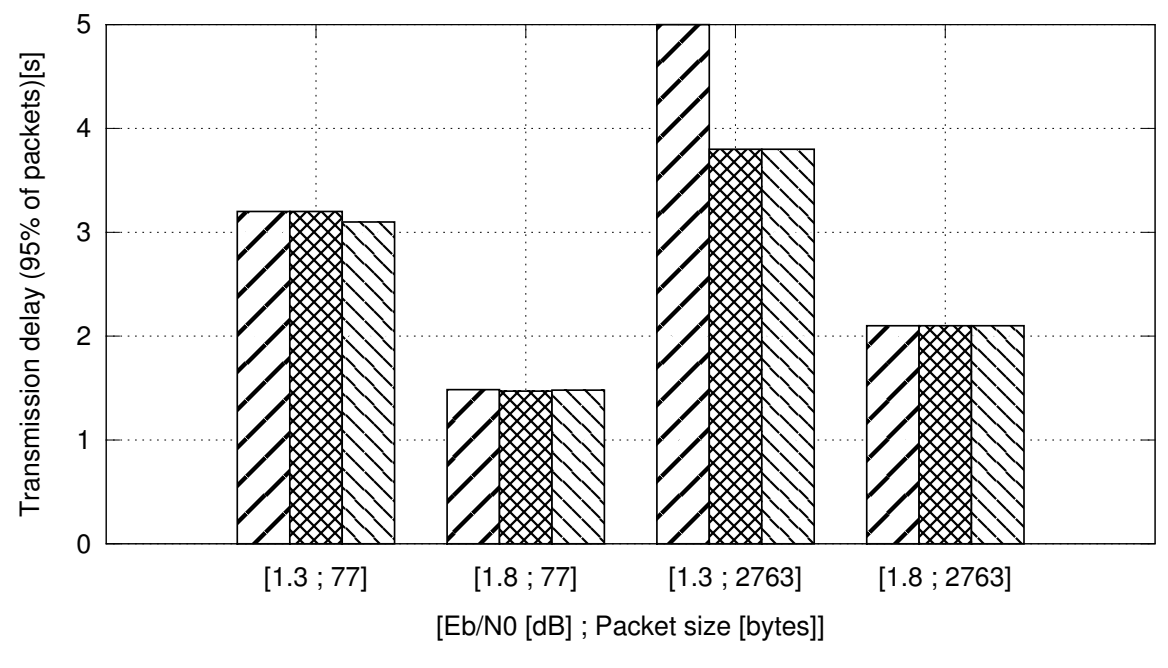

no $\mathrm{ARQ} / \mathrm{HARQ}$

$A R Q \$ 8 \times 8 \times 8$

HARQ $\triangle I \square$

Fig. 3. Transmission delay of $95 \%$ of the packets

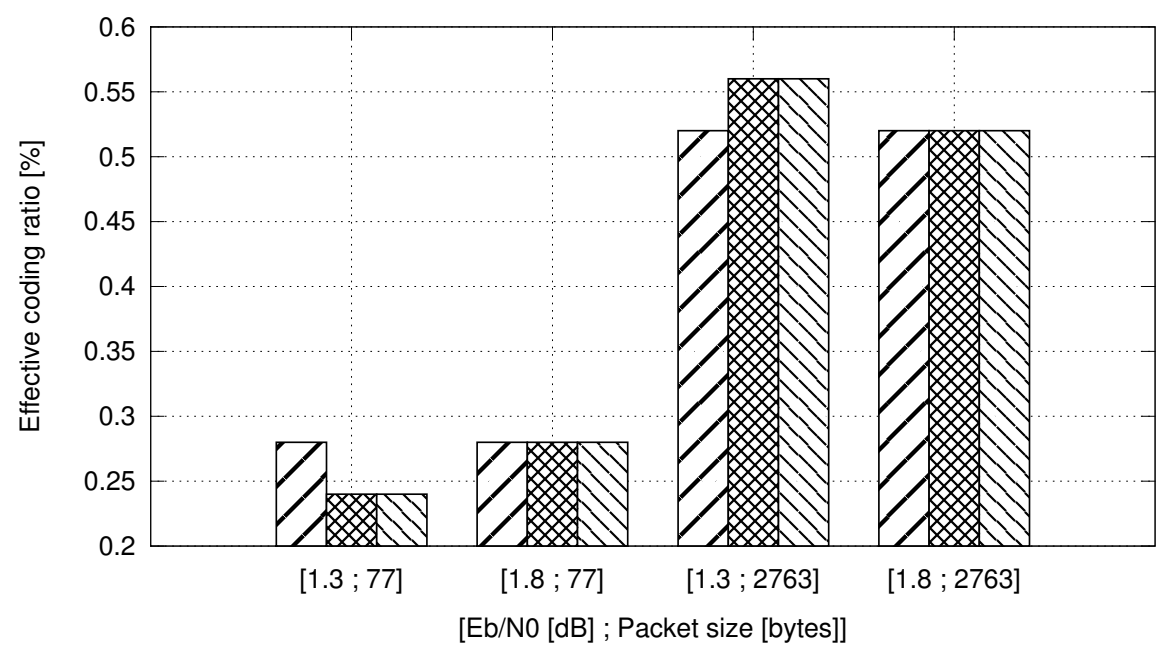

no ARQ/HARQ $\square \triangle$ ARQ

Fig. 4. Effective coding ratio

that this specific case is enough to justify the need for retransmission at the link layer when dealing with high PER at link layer.

However, as we discussed in Section 2.4, Figure 4 shows that in this example (large packets in high PER conditions), in order to decrease the transmission de- 
lay, more capacity is exploited to transmit one single data packet thus increasing the risk of jamming the system in high load conditions.

As shown, in some specific use cases, link layer retransmissions are beneficial. This experiments also show that this link layer retransmissions have a cost in terms of capacity usage. However, results of this section do not highlight differences between ARQ and HARQ link layer retransmissions schemes, which we focus on in Section 5.2.

\subsection{Comparison of ARQ and H-ARQ on TCP performance}

The results presented in Section 5.1 do not reveal any advantage for or against ARQ and HARQ implementations at the link layer. We present additional simulations in this section, with a higher PER at physical layer and considering various size of applicative packets. We plot in Figure 5 the transmission delay of $95 \%$ of the packets and in Figure 6, the effective coding ratio. As we compare ARQ and HARQ, we focus on a specific implementation of HARQ. HARQ does not transmit a first FEC block, but introduce more additional retransmission packets than ARQ (more details can be found in Section 2.3).

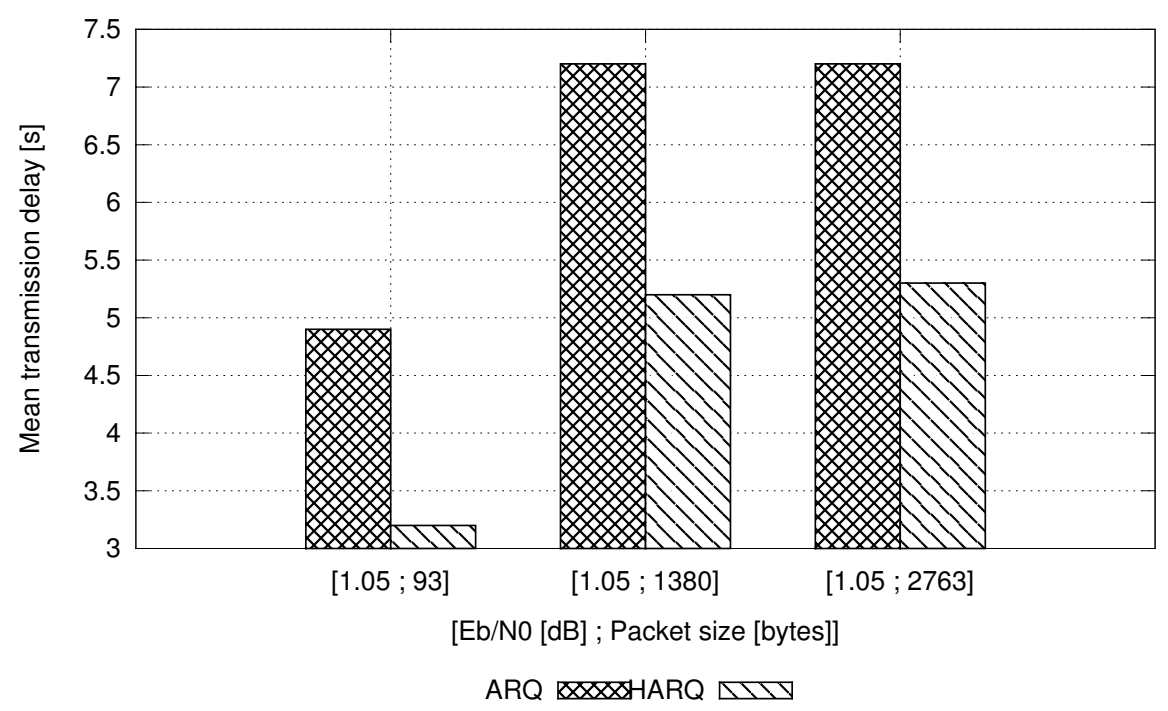

Fig. 5. Transmission delay of $95 \%$ of the packets

We measure that HARQ can reduce the transmission delay of ARQ on several cases (in Figure 5), making their use of interest in case of a very noisy channel. However, this improvement has a cost in terms of capacity usage as illustrated in Figure 6. 


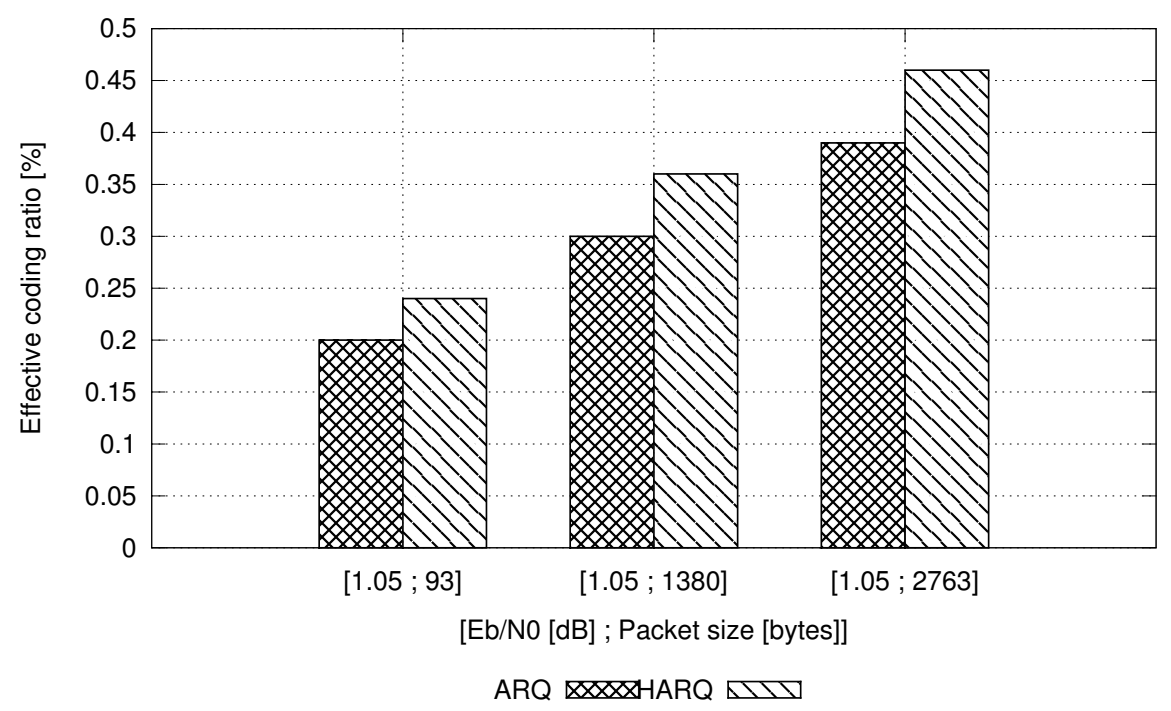

Fig. 6. Effective coding ratio

Considering a specific HARQ reduces the scope of our results, but we highlight the need for deeper studies on the impact of the parameters of HARQ on the TCP performance in the context of critical aeronautical communications. We illustrate that different link layer retransmission schemes have different performances in terms of delay and capacity utilization, illustrating the discussion presented in Section 2.4.

\subsection{Limits of interpretations}

The considered physical layer performances are located well below the currently defined targets for satellite communication systems used in aeronautical communications. For systems which are well designed, and for which the physical layer performances meet the established criterion, the use of ARQ and HARQ at the link layer does not provide any improvement when compared to the use of TCP without any link layer retransmissions.

Furthermore, the use of SWIM like communication paradigms, heavily relying on protocols such as HTTP and SOAP make the use of TCP a natural choice. In these contexts, although the introduction of ARQ and HARQ can improve the performance in high PER contexts, the overall transmission delays observed (even in a fully optimized context) are not compliant with the application requirements. Finally, the presence of timeout values in protocols executing above TCP have not been considered in this study. 


\section{Conclusion}

In this article, we argue for the introduction of link layer retransmissions schemes in the context of aeronautical communications. We make use of link layer simulation traces on which we simulate link layer retransmissions to assess the impact on ARQ and HARQ on the performance of TCP. We show that, in worst cases with regards to the propagation channel conditions, link layer retransmissions enable to transmit applicative data in acceptable delays for the safety of aeronautical communications. We also illustrate that a trade-off must be found between having a low transmission delay and a good channel capacity usage.

\section{Acknowledgments}

This work presented in this article documents part of the simulation scenarios and results obtained in a study funded by CNES in which TéSA and Thales Alenia Space took part.

\section{References}

1. S. Lin and D. J. Costello, Error control coding: fundamentals and applications, vol. Chapter 15. Ed. Prentice-Hall, 1983.

2. E. Berlekamp, R. Peile, and S. Pope, "The application of error control to communications," Communications Magazine, IEEE, vol. 25, pp. 44-57, april 1987.

3. J. Kurose and K. Ross, "A top-Down Approach," in Computer Networking, p. 284, Ed. Addison Wesley, 2008.

4. J. Postel, "User Datagram Protocol," RFC 768, RFC Editor, Aug. 1980.

5. C. Callegari, S. Giordano, M. Pagano, and T. Pepe, "Behavior analysis of tcp linux variants," Comput. Netw., vol. 56, pp. 462-476, Jan. 2012.

6. M. Allman, V. Paxson, and E. Blanton, "TCP Congestion Control," RFC 5681, RFC Editor, Fremont, CA, USA, Sept. 2009.

7. W. Chauvet, C. Amiot-Bazile, and J. Lacan, "Prediction of performance of the dvb-sh system relying on mutual information," in Advanced satellite multimedia systems conference (asma) and the 11th signal processing for space communications workshop (spsc), 2010 5th, pp. $413-420$, sept. 2010.

8. N. Kuhn, E. Lochin, J. Lacan, R. Boreli, C. Bes, and L. Clarac, "Enabling realistic cross-layer analysis based on satellite physical layer traces," in PIMRC'12, pp. 285290, 2012.

9. N. Kuhn, E. Lochin, J. Lacan, R. Boreli, C. Bes, and L. Clarac, "Clift: a crosslayer information tool to perform cross-layer analysis based on real physical traces," CoRR, vol. abs/1206.5459, 2012. 